\title{
Exciton-Exciton Annihilation as a Mechanism for Uphill Transfer in a Molecular Excitonic System
}

\author{
Craig N. Lincoln ${ }^{1}$, Matthias Block ${ }^{2}$, Bastian Baudisch ${ }^{2}$, Pavel Malevich ${ }^{1}$, Hans von \\ Berlepsch $^{3}$, Eberhard Riedle ${ }^{2}$, and Jürgen Hauer ${ }^{4, *}$ \\ ${ }^{1}$ Photonics Institute, Vienna University of Technology, Gusshausstrasse 27, 1040 Vienna, Austria \\ ${ }^{2}$ Lehrstuhl für BioMolekulare Optik, Ludwig-Maximilians-Universität, Oettingenstr. 67, 80538 \\ München, Germany \\ ${ }^{3}$ Forschungszentrum für Elektronenmikroskopie, Institut für Chemie und Biochemie, Freie \\ Universität Berlin, Fabeckstrasse 36a, D-14195 Berlin, Germany \\ ${ }^{4}$ Professur für Dynamische Spektroskopien, Fakultät für Chemie, Technische Universität München, \\ Lichtenbergstr. 4, D-85748, Garching b. München, Germany
}

\begin{abstract}
Exciton dynamics in a HJ-aggregate of cyanine dye TTBC are investigated by transient absorption with a time resolution of $<60 \mathrm{fs}$ and power-dependent emission spectroscopies. Both measurements are consistent with an exciton delocalization length of $\sim 28$ monomers. A model assuming diffusive exciton motion reveals that the exciton mobility is at least bimodal and restricted to one spatial dimension. J-band diffusion rates of 2.69 and $2.79 \mathrm{e}^{-3} \mathrm{~cm}^{2} \mathrm{~s}^{-1}$ are found, leading to maximal diffusion lengths of 449 and $14.5 \mathrm{~nm}$. The findings indicate that exciton-exciton annihilation is the origin of effective uphill transfer. This mechanism, if present under solar radiation, maybe useful for organic photovoltaic systems.
\end{abstract}

The study of excitonic diffusion in organic semiconductors is highly relevant to applications such as artificial light harvesting and organic light emitting diodes. High exciton mobility is a key characteristic for the efficient operation of such devices and is determined by several processes, including exciton-exciton annihilation (EEA) [1]. EEA is the deactivation of excitons by the fusion of two followed by non-radiative (NR) relaxation (Fig. 1g). Here we discuss results on a strongly coupled molecular system, namely a HJaggregate of a cyanine derivative 5,5',6,6'tetrachloro-1,1',3,3'-tetraethylbenzimidacarbocyanine (TTBC-Cl) [2,3]. TTBC-Cl exhibits characteristically efficient excited state energy transport (EET) due to the aggregate's high degree of order leading to barrier-free excitonic diffusion. The sample exhibits a large absorption cross section and superradiant emission (see Fig. 1a). These characteristics have led to the use of aggregates as FRET donors and acceptors in applications such as light harvesting, photoreduction, photovoltaics, sensors and biomedical imaging [4]. According to cryo-transmission electron microscopy, TTBC-Cl forms single-walled tubes with a diameter of only $35 \AA$ and lengths

* Corresponding author: juergen.hauer@tum.de 


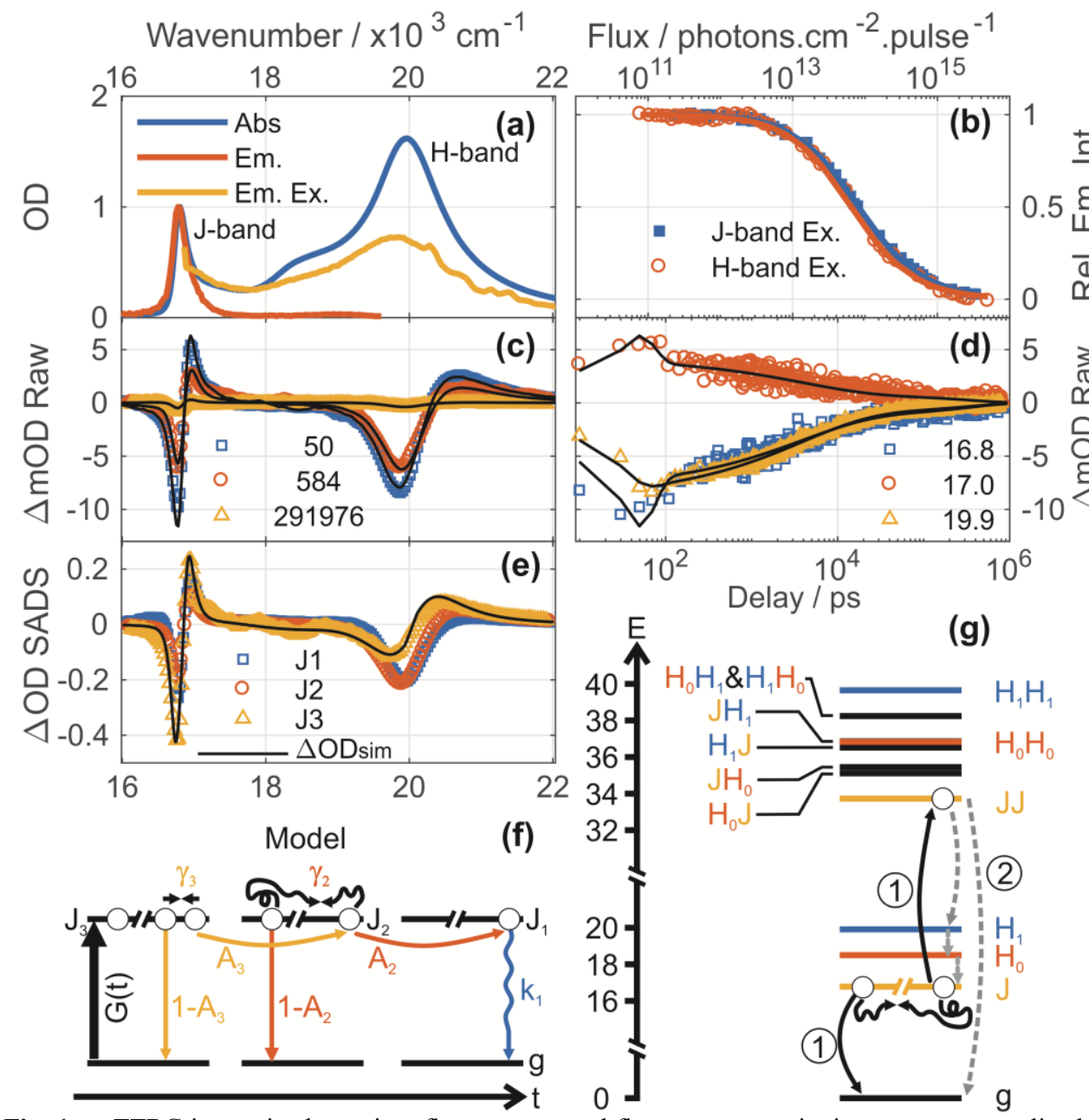

Fig. 1. a. TTBC isotropic absorption, fluorescence and fluorescence excitation spectra normalized to $100 \%$ conversion at $\sim 17,000 \mathrm{~cm}^{-1}$. b. Power-dependent emission intensity for J- and H-band excitation (square and circle). c. Transient absorption spectra for J-band excitation and horizontal probe at $50 \mathrm{fs}, 584 \mathrm{fs}$ and $292 \mathrm{ps}$ (square, circle, triangle). d. associated time traces to $\mathbf{c}$ at 16,800, 17,000 and $19,900 \mathrm{~cm}^{-1}$, corresponding to J-band bleach, excited state absorption and H-band bleach, respectively (square, circle, triangle). e. Retrieved state associated difference spectra (SADS) for a global fit to transient absorption and power-dependent fluorescence (solid lines in $\mathbf{b}, \mathbf{c}$ and $\mathbf{d}$ ) data using the model depicted in $\mathbf{f}$. $\triangle$ ODsim is an ideal difference spectra for pure J-band population, clearly, states $\mathrm{J}_{2}$ and $\mathrm{J}_{1}$ have additional positive signal to the blue of the $\mathrm{J}$-band and additional $\mathrm{H}$-band bleach, ascribed to H-band excited state population. f. Model used to globally fit TA and PDEm. data (See text for details). g. TTBC energy level diagram estimated from absorption and transient absorption data. Depiction of EEA processes of fusion (1) and relaxation to either lower states or ground (2).

in the $\mu \mathrm{m}$ range [3]. Recently, a molecular structure was suggested, consistent with these dimensions and the absorption and LD spectra [5]. The structure is helical, formed by rings of six monomeric units with transition dipoles aligned $74^{\circ}$ to the longitudinal aggregate axis, and an interring slip of $30^{\circ}$. We show using femtosecond transient absorption (TA) and power-dependent emission spectroscopies that the rate of EEA in the TTBC-Cl 
aggregate is at least biphasic and can lead to the population of energetically higher lying states, i.e. 'uphill transfer'.

TTBC's absorption spectrum consists of at least three absorption bands, however, we will concentrate on the two most extreme bands, namely, the narrow, red-shifted with respect to the monomer and polarized parallel to TTBC's long axis J-band near $17,000 \mathrm{~cm}^{-1}$ and the broad, blue shifted and perpendicularly polarized H-band near $20,000 \mathrm{~cm}^{-1}$ (Fig. 1a). Using narrowband excitation we selectively excited the $\mathrm{J}$ - or $\mathrm{H}-$ bands of an aligned sample of TTBC (long axis vertical) and measured the power-dependent emission and transient absorption probed with white-light polarized either vertically or horizontal to the aggregate. Global fitting of the power-dependent emission (Fig. 1b) and vertically polarized J- or H-band pumped data reveals TTBC has an exciton delocalization length of $\sim 28$ monomers, diffusion is restricted to one dimension and the mobility is at least bimodal. The $1 \mathrm{D}$ diffusion lengths were found to be 449 and $14.5 \mathrm{~nm}$. For more details we refer readers to ref [6].

Focusing on uphill transfer, we analyze the J-band pumped transient absorption probed with horizontal polarization to preference the H-band response (Figs. 1c and d). The data were globally fitted using the model depicted in figure $1 \mathrm{f}$ to retrieve state associated difference spectra (SADS; Fig. 1e) for the three states $J_{3}-J_{1}$ included in the model. $J_{3}$ is the first excited state ascribed to biexcitonic to the JJ-band in figure $1 \mathrm{~g}$ and where relaxation is modelled as EEA. The retrieved $\mathrm{J}_{3}$ SADS lineshape is consistent with a purely J-band excited state population as exemplified by the simulated difference spectra ( $\Delta$ ODsim; fig. 1e) that is constructed from a summation over a negative absorption spectrum (GSB), a negative J-band fluorescence spectra (SE) and a blue shifted by $170 \mathrm{~cm}^{-1}$ positive absorption spectra (ESA). Note the near $(\mathrm{GSB}+\mathrm{SE})$ :ESA ratio of 2:1 for the J-band characteristic of its superradiant nature.[2] The lineshape of the subsequent states, $\mathrm{J}_{2}$ and $\mathrm{J}_{1}$, differ significantly from the purely J-band simulated difference spectra with features consistent with H-band character [2]. Specifically, the increase of ESA to the red of the Jband and the apparent loss of J-band bleach are indicative of additional H-band to HJ-band ESA, while the increased bleach near $20,000 \mathrm{~cm}^{-1}$ is ascribed to an increase in H-band SE. As the build-up of $\mathrm{H}$-band population is delayed, i.e. absent in state $\mathrm{J}_{3}$, appearing in the 100-300 fs range we ascribe EEA as the mechanism of uphill transfer, as depicted in figure $1 \mathrm{~g}$. Moreover, the persistence of the $\mathrm{H}$-band character in states $J_{2}$ and $J_{1}$ suggests at least part of this H-band population does not rapidly relax to the J-band as would be expected [2], which is consistent with the lower fluorescence QY observed for the H-band (Fig. 1a). One possible explanation is competing aggregate structures in the current preparation each with different band structures. A topic of future work.

\section{References}

1. O.V. Mikhnenko, P.W.M. Blom, T.Q. Nguyen, Energy Environ. Sci., 8, 1867-1888 (2015).

2. M. Bednarz, J. Knoester, J Phys Chem B, 105, 12913-12923 (2001).

3. H. von Berlepsch, C. Bottcher, Langmuir, 29, 4948-4958 (2013).

4. J.L. Bricks, Y.L. Slominskii, I.D. Panas, A.P. Demchenko, Methods Appl Fluores, 6, (2018).

5. C. Friedl, T. Renger, H.V. Berlepsch, K. Ludwig, M.S.A. Busch, J. Megow, J Phys Chem C, 120, 19416-19433 (2016).

6. C.N. Lincoln, M. Block, B. Baudisch, H. von Berlepsch, E. Riedle, J. Hauer, Angew. Chem., (to be published). 